Reprod. Nutr. Dévelop., 1982, 22 (2), 325-344.

\title{
Quelques aspects des interactions hormonales entre la mue et la vitellogenèse chez le Crustacé Amphipode Orchestia gammarellus (Pallas) (')
}

\author{
Marie-Françoise BLANCHET-TOURNIER
}

Laboratoire de Sexualité et Reproduction des Invertébrés, ERA 409

Université Pierre et Marie Curie, Bât. A, 4, place Jussieu, 75230 Paris Cedex 05.

Summary. Some aspects of hormonal interactions between moiting and vitellogenesis in Orchestia gammarellus (Pallas) (Crustacea, Amphipoda).

The mechanisms which coordinate and regulate secondary vitellogenesis and molting in Orchestia gammarel/us have been studied in natural and experimental conditions. The onset of secondary vitellogenesis, requiring a protocerebral factor and a low titer of ecdysteroids, occurs only after normal folliculogenesis. The secondary follicular tissue would secrete vitellogenin stimulating ovarian hormone (VSOH) which stimulates the subepidermal adipose tissue to produce vitellogenin. $\mathrm{VSOH}$ and ecdysteroids are indispensable to normal vitellogenin synthesis during secondary vitellogenesis. Moreover, the $\mathrm{Y}$-organ controls vitellogenin entry into the oocytes.

In secondary vitellogenesis the ovary would stimulate the secretion of molt inhibiting hormone $(\mathrm{MIH})$ which would act on the epidermis, rendering this tissue unresponsive to the stimulatory action of ecdysterone.

The $Y$-organ and the protocerebrum are involved in the control of the molting processes independently of their action on vitellogenesis.

\section{Introduction.}

Contrairement aux femelles d'Insectes, la plupart des femelles de Malacostracés continuent à muer après la puberté. Différentes relations, d'ordre chronologique, entre la mue et la reproduction ont été observées. Chez certaines espèces, la vitellogenèse secondaire se réalise en un intermue. C'est le cas d'Orchestia gammarellus (Charniaux-Cotton, 1957), des 2 Isopodes: Porcellio dilatatus (Legrand, 1958) et /dotea balthica (Reidenbach, 1971), de divers Décapodes, notamment Lysmata sedicaudata (Charniaux-Cotton et Touir, 1973). Chez d'autres espèces, la vitellogenèse secondaire dure plusieurs intermues. Les Isopodes Asellus aquaticus (Balesdent, 1964) et Ligia oceanica (Besse, 1976) font

(1) Cet article est le résumé d'une partie d'une thèse de Doctorat d'Etat soutenue à I'Université $P$. et M. Curie (Paris VI) le 25 septembre 1980. 
partie de ce cas. Quant aux Décapodes Palaemon serratus (Drach, 1955) et Atyaephyra desmaresti (Descouturelles, 1978), la vitellogenèse secondaire se déroule en un ou plusieurs intermues suivant les saisons.

Si pour l'ensemble de ces Crustacés, la croissance ovocytaire s'achève en même temps que l'intermue, la ponte ayant lieu après l'exuviation, ce n'est pas le cas de Carcinus maenas. En effet, chez ce crabe, la vitellogenèse secondaire se réalise pendant le stade $\mathrm{C}_{4}$ (Demeusy, 1963) et la ponte survient avant la prémue (Cheung, 1966).

O. gammarellus représente un modèle simple de synchronisme muevitellogenèse. Cet Amphipode se reproduit au printemps et en été (soit de mars à octobre). Durant cette phase d'activité génitale, la vitellogenèse secondaire et l'intermue sont étroitement liées et l'exuviation est suivie de ponte. Le texte qui suit résume les données actuellement acquises chez la femelle pubère d'O. gammarellus concernant les déroulements synchrones ou asynchrones du cycle de mue et du cycle ovarien dans diverses conditions naturelles ou expérimentales. Plusieurs mécanismes régulateurs liant la croissance et la reproduction sont dégagés de cette étude.

\section{Synchronisme mue-vitellogenèse chez 0 . gammarellus. Description.}

\section{A. Cycle de mue et durée des différents stades chez la femelle pubère en vitellogenèse.}

Seule la femelle pubère en reproduction présente une durée stricte des stades d'intermue et donc du cycle de mue : $23-24$ jours à $20^{\circ} \mathrm{C}$.

\section{PLANCHE I}

Coupes transversales d'ovaires de femelles d'O. gammarellus expérimentales et organe $Y$ chez une femelle témoin.

1. Ovaire d'une femelle privée de la région antéro-médiane du protocérébron en fin $\mathrm{D}_{2}$, soit $24 \mathrm{~h}$ avant l'exuviation, et disséquée en début $\mathrm{C}_{\mathrm{c}}, 16$ jours plus tard. Noter le resserrement des cellules folliculaires (1) (leur hauteur peut atteindre $12 \mu \mathrm{m}$ ) et leur élongation (2) en vue de former un épithélium autour de cet ovocyte qui va entrer en vitellogenèse secondaire.

2. Un des 2 massifs cellulaires constituant les organes $Y$ (coupe transversale de tête). Limitée par une basale, cet organe est accolé par sa base à l'épiderme.

3. Ovaire d'une femelle privée de ses organes $Y$ au début de la vitellogenèse secondaire (début $C_{b}$ ) et bloquée à ce stade depuis 10 jours. La taille de l'ovocyte (environ $220 \mu \mathrm{m}$ ), l'état du cytoplasme (notamment la présence de globules vitellins en périphérie) et la position centrale de la vésicule germinative correspondent à l'état d'un ovocyte de femelle témoin en $\mathrm{C}_{b}$.

4. Ovaire d'une femelle privée de la région antéro-médiane du protocérébron au cours de la vitellogenèse secondaire $\left(\mathrm{D}_{0}\right)$ et bloquée à ce stade de mue depuis 13 jours; I'ovocyte présente toutes les caractéristiques d'une fin de vitellogenèse : présence de plaquettes vitellines et de globules lipidiques dans tout le cytoplasme, vésicule germinative en fin de migration, taille d'environ $650 \times 520 \mu \mathrm{m}$.

c, cuticule ; e.f., épithélium folliculaire ; ep, épiderme ; g.l., globule lipidique ; g.v., globule vitellin ; m., mitose de cellule folliculaire ; o.f. v.p., ovocyte en fin de vitellogenèse primaire ; o.f.v.s., ovocyte en fin de vitellogenèse secondaire; o.pv., ovocyte en prévitellogenèse ; o.r., ovocyte en résorption; o.v.s., ovocyte en vitellogenèse secondaire; p.v., plaquette vitelline; v.g., vésicule germinative ; $Y$, organe $Y ; z . g$., zone germinative 

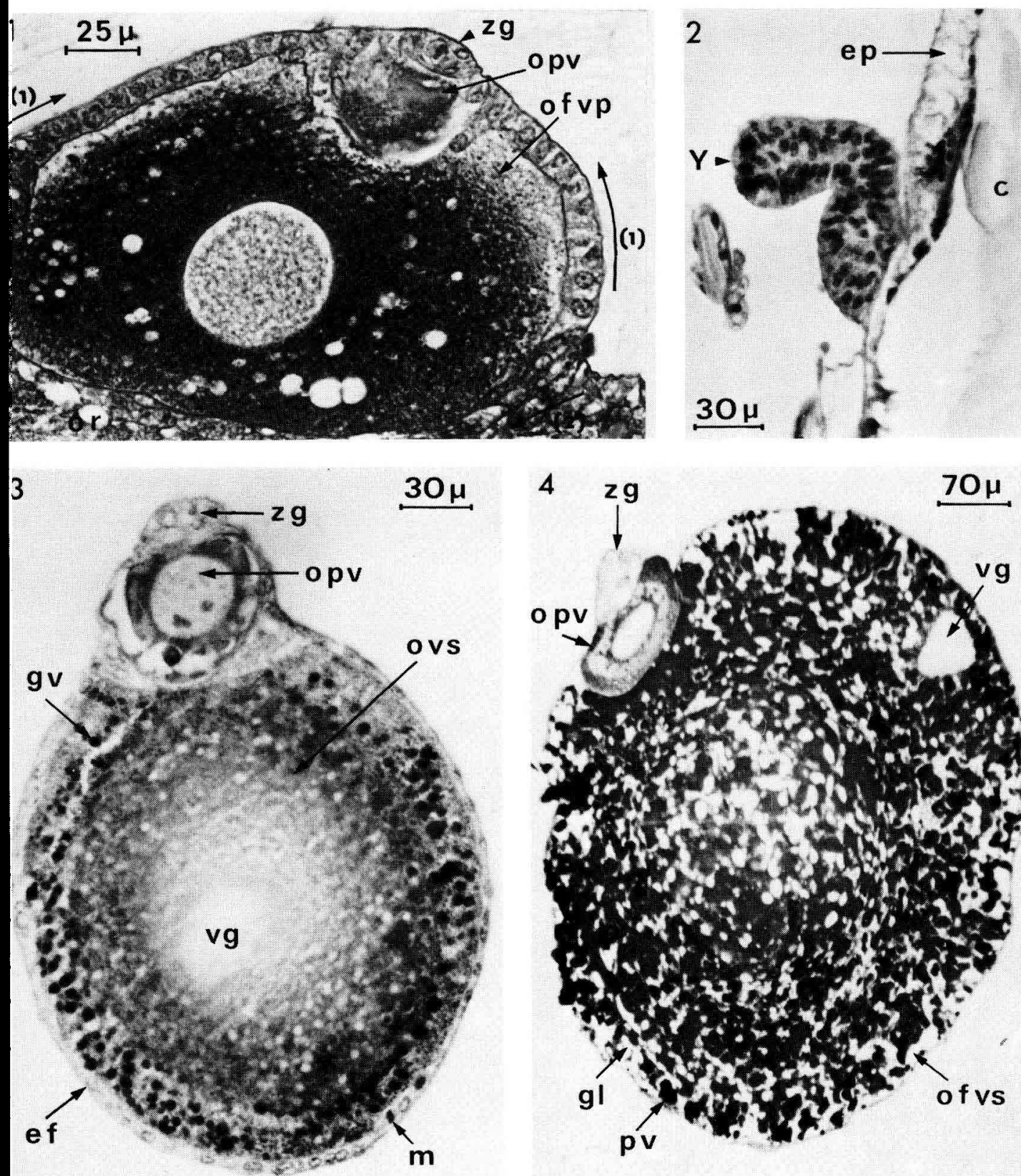
La postmue (= postecdysis) comprend 2 périodes $A$ et $B(A=12 \mathrm{~h}$; $B=2$ jours). Elle est caractérisée par l'achèvement de l'exosquelette : sécrétion des couches cuticulaires post-exuviales et minéralisation de la cuticule.

La période $C$ (= diecdysis) dure 9 jours. Elle débute par un décollement de l'épiderme de l'ancienne cuticule au niveau du dactylopodite des péréiopodes (étape $C_{a}=3-4$ jours). La matrice ainsi rétractée s'invagine (étape $C_{b}=3$ jours) et se recouvre d'un étui cuticulaire (étape $C_{c}=2-3$ jours). Cette formation précoce des ongles dactyliens est un phénomène exceptionnel chez les Crustacés.

La prémue (proecdysis) se déroule en 12 jours. Elle débute par l'étape $D_{0}$ (durée : $4-5$ jours). Cette étape, dès son amorce, est diagnostiquée par le décollement de l'épithélium dans l'angle antéro-distal du propodite; cette apolyse s'étend à l'ensemble de l'animal dans les jours qui suivent. L'étape $D_{1}$, (3 jours), subdivisée en 3 stades $\left(D_{1^{\prime} a}, D_{1^{\prime} b}, D_{1^{\prime} c}\right)$, correspond à la formation de la fente circulaire des matrices trichogènes. L'étape $D_{2}$ (4-5 jours) est marquée par le dépôt de couches cuticulaires préexuviales ; elle précède l'exuviation.

\section{B. Cycle de vitellogenèse.}

L'ovaire d'O. gammarellus et l'ensemble des phénomènes qui contribuent à réaliser une vitellogenèse normale ont fait l'objet d'un grand nombre de travaux (voir revues Charniaux-Cotton, 1973, 1975, 1978, 1980 ; Meusy, 1980 ; Zerbib, 1980). La vitellogenèse comporte deux phases bien distinctes:

- la vitellogenèse primaire ou endogène caractérisée par l'activité de synthèse du réticulum endoplasmique granulaire, conduisant à l'accumulation de vitellus protéique dans les ovocytes (Zerbib, 1980) :

- la vitellogenèse secondaire due à une entrée massive de vitellogénine par micropinocytose et à une accumulation de nombreux globules lipidiques dans l'ooplasme (Zerbib, 1980). Cette deuxième phase n'a lieu que durant la saison de reproduction.

Nous mettrons l'accent sur les phénomènes de vitellogenèse secondaire qui sont en relation avec le cycle de mue.

L'exuviation s'étant réalisée, la ponte n'a lieu que s'il y a eu accouplement. Dans le cas contraire, les ovocytes mûrs sont rapidement résorbés. Quoi qu'il en soit, l'ovaire entre dans un nouveau cycle de vitellogenèse.

En postmue, des remaniements importants se produisent en ce qui concerne les relations cellules mésodermiques-ovocytes dans l'ovaire. Des observations récentes de coupes histologiques d'ovaires de femelles privées de la région antéro-médiane du protocérébron (le démarrage de la vitellogenèse secondaire est fortement ralenti ; cf. plus loin) apportent des indications sur leur mise en place autour des ovocytes (PI. I, 1). Des déformations cellulaires sont visibles et témoignent du déplacement des cellules mésodermiques qui viennent entourer les ovocytes en fin de vitellogenèse primaire pour former l'épithélium folliculaire secondaire. Dès que ce dernier est mis en place, l'ovocyte émet des macrovillosités en direction des cellules folliculaires et amorce la vitellogenèse secondaire. Ces phénomènes de folliculogenèse secondaire (Charniaux-Cotton, 1974, 1978) s'achèvent au cours du stade $C_{a}$ de l'intermue. 
De $C_{a}$ à la fin de l'intermue, la taille des ovocytes ne va cesser de croître (fig. 1, a). De $0,03 \mathrm{~mm}^{3}$ en fin de vitellogenèse primaire, l'ovocyte atteint un volume de $1,06 \mathrm{~mm}^{3}$ en fin de vitellogenèse secondaire, soit environ 35 fois le volume initial. L'accumulation de matériel lipo-glyco-caroténoprotéique d'origine exogène, et de lipides, notamment de triglycérides assure cette croissance ovocytaire (Zerbib, 1976).

La vésicule germinative, tout d'abord centrale, va migrer vers la périphérie de l'ovocyte au stade $D_{0}$ ou $D_{1}$ 'a. La rupture de la membrane nucléaire qui marque la reprise de la méiose aura lieu obligatoirement lors de l'exuviation (MathieuCapderou, 1980).

A la fin de l'intermue, soit en fin $D_{2}$, les ovocytes cessent de croître ; les macrovillosités, à leur surface, se rétractent (Zerbib, 1973). L'ovocyte perd son étroit contact avec l'enveloppe folliculaire. La disparition des septa, cloisons séparant les ovocytes, permet la ponte (Mathieu-Capderou, 1980).

L'incubation des œufs a lieu dans le marsupium (sorte de poche formée par la paroi ventrale de la femelle d'une part et les oostégites d'autre part ; les soies ovigères assurant une bonne fermeture). L'éclosion des jeunes s'effectue au stade $D_{0}$, soit une douzaine de jours après la ponte. Les petits restent plusieurs jours dans la poche incubatrice avant de se séparer de leur mère.

\section{Activité endocrine cyclique de /'ovaire d'O. gammarellus ?}

Des ovariectomies et des implantations d'ovaires chez des femelles d'O. gammarellus castrées ont permis de montrer que les soies ovigères sont sous le contrôle d'une hormone provenant de l'ovaire en vitellogenèse (Charniaux-Cotton, 1957). Cette hormone induit l'étirement maximum des matrices trichogènes des oostégites lors des stades $D_{1}$ (Charniaux-Cotton, 1957). Cette sécrétion se renouvelle à chaque cycle de mue puisque lors de l'arrêt de l'activité génitale, en automne, on note la formation de soies juvéniles ou intermédiaires.

Des expériences comparables ont conduit à postuler l'existence d'une VSOH (Vitellogenin Stimulating Ovarian Hormone), hormone ovarienne contrôlant la synthèse de la vitellogénine (Junéra et al., 1977), hétéro-protéine circulant dans I'hémolymphe des femelles en reproduction. La synthèse de la vitellogénine par le tissu adipeux sous-épidermique (Junéra et Croisille, 1980; Croisille et Junéra, 1980) présente des variations au cours du cycle de mue (Meusy et al., 1974) : faible sinon nulle en postmue, elle augmente au cours du stade $C_{a}$ pour atteindre un maximum en $C_{c}-D_{0}$; elle diminue durant le stade $D_{2}$ soit dans les jours qui précèdent l'exuviation (fig. 1 , b).

Qu'il s'agisse d'une seule hormone ou de 2 hormones distinctes contrôlant les soies ovigères et la synthèse de la vitellogénine, les cellules folliculaires apparaissent comme le seul site de synthèse possible (Charniaux-Cotton, 1957, 1978).

Enfin, la possibilité pour l'ovaire d'O. gammarellus de synthétiser des ecdystéroïdes reste à démontrer. Les ovaires de cette espèce contiennent des ecdystéroïdes durant tout leur développement : le taux hormonal ovarien varie peu jusqu'en $D_{1}$, (de 20 à 30 pg/ovaire détectés en RIA) et augmente en fin de vitellogenèse, soit en $D_{2}$ (70 pg/ovaire, ce qui représente 3 p. 100 des ecdystéroïdes 
totaux) (Blanchet et al., 1979; fig. 1, c). A noter que cette augmentation est synchrone des pics d'ecdystéroïdes circulants et tissulaires (Blanchet et al., 1979 ; fig. 1, d et e) ce qui n'est pas le cas des autres Crustacés étudiés. En effet, chez le Crabe Acanthonyx lunulatus qui réalise la vitellogenèse après la mue de puberté, deux pics d'ecdystéroïdes sont observés dans les ovaires au début et à la fin de la vitellogenèse alors que ces mêmes hormones sont absentes dans I'hémolymphe (Chaix et de Reggi, 1979). De même, chez Carcinus maenas, le taux $d^{\prime}$ ecdystérö̈des ovariens augmente au cours de la vitellogenèse tandis que le taux sanguin reste faible et est inférieur au $1 / 10$ de la concentration minimale trouvée dans les ovaires (Lachaise et Hoffmann, 1977). L'ablation des organes $Y$ n'empêchant pas l'augmentation du taux d'ecdystéroödes contenus dans les ovaires, ces mêmes auteurs admettaient la possibilité pour la gonade de synthétiser

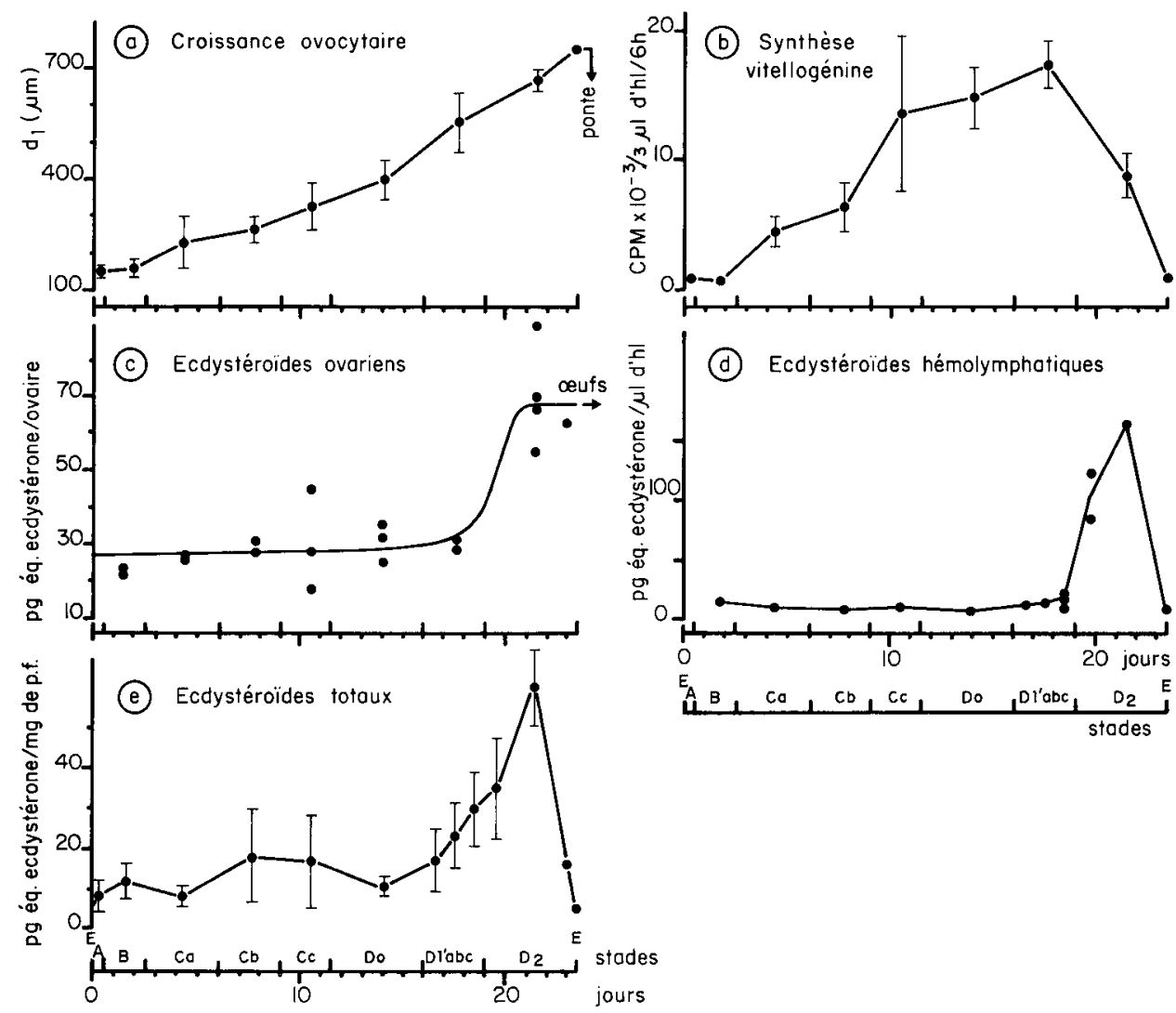

FIG. 1. - Comparaison des variations de la croissance des ovocytes en vitellogenèse secondaire (moyennes $\pm t$.ES : $t$, variable de Student, pour $n-1$ degrés de liberté et un risque de 0,05 ) (d'après Blanchet-Tournier, 1980), de la synthèse de la vitellogénine (moyennes $\pm E S$ ) (d'après Junéra, 1981) et des ecdystérö̈des ovariens, circulants et tissulaires (d'après Blanchet et al., 1979) au cours $d^{\prime} u n$ cycle de mue chez la femelle $d^{\prime} O$. gammarellus en période de reproduction. En abscisses, les stades d'intermue et leur durée en jours. $E$ : exuviation. $d_{1}$ : moyenne pour 1 ovaire du plus grand diamètre mesuré sur des montages in toto. 
l'ecdysone. Cette hypohèse a été récemment vérifiée : l'ovaire de Carcinus maenas est capable de synthétiser de l'ecdysone, de l'ecdystérone et de la ponastérone A à partir du cholestérol (Lachaise, 1981). Bien que l'absence de cholestérol libre et de ses esters ait été signalée par histochimie chez plusieurs espèces de Décapodes (Brodzicki, 1963), on ne peut donc exclure l'ovaire comme site de biosynthèse d'ecdystéroïdes chez les Crustacés comme c'est le cas chez les Insectes.

A la (Aux) synthèse(s) hormonale(s) citée(s) ci-dessus, s'ajouterait ( ${ }^{1}$ ) alors une production cyclique d'ecdystéroïdes par les cellules folliculaires. Les ecdystéroïdes, stockés dans les ovocytes, et retrouvés dans les œufs après la ponte participeraient à l'élaboration des embryons.

\section{Etude expérimentale des interactions entre la mue et la vitellogenèse chez o. gammarellus.}

Le synchronisme étroit observé entre la mue et la vitellogenèse secondaire chez 0 . gammarellus implique des interactions entre ces 2 phénomènes. La recherche de ces interactions peut être envisagée en supprimant l'un de ces événements directement ou par l'intermédiaire de(s) l'organe(s) ou de(s) l'hormone(s) qui le régule(nt).

A. Etude $d u$ déroulement $d u$ cycle de mue en absence de vitellogenèse secondaire. - L'absence de vitellogenèse secondaire chez des femelles pubères d'O. gammarel/us peut être naturelle (cas du repos génital hivernal) ou expérimentale (ovariectomie, augmentation expérimentale du taux d'ecdystérone). Voyons ces différents cas :

1. Cas des femelles en repos sexuel. - Durant le repos sexuel hivernal, seule la vitellogenèse primaire se réalise. Les ovocytes, bien que capables d'accumuler quelques réserves, ne dépasseront pas une taille de $250 \mu \mathrm{m}$. Ce blocage de la croissance ovocytaire est probablement dû à l'absence de synthèse de vitellogénine pendant toute cette période (Meusy et al., 1974). La durée du cycle de mue chez ces femelles est plus courte que chez les femelles en reproduction : 19 jours en moyenne au lieu d'une durée stricte de 23-24 jours (Blanchet et CharniauxCotton, 1971). Ce sont les étapes $C, D_{0}$ et $D_{2}$ qui subissent un léger raccourcissement. A noter cependant que pour un même stade de mue, le taux d'ecdystéroïdes n'est pas significativement différent de celui observé chez les femelles en vitellogenèse (Blanchet et al., 1979).

2. Ovariectomie. - L'ovariectomie chez des femelles d'O. gammarellus en reproduction entraîne une diminution égale aux $2 / 3$ environ de la durée de l'intermue (Charniaux-Cotton, 1957). L'auteur note que cette accélération semble due à l'absence de vitellogenèse. En effet, lors de la reprise de l'activité génitale au printemps chez $O$. gammarellus, la durée du cycle de mue passe brusquement de 15 jours (intermue sans vitellogenèse) à 22 jours (intermue avec $1^{\text {re }}$ vitellogenèse).

3. Augmentation expérimentale du taux d'ecdystérone. - Un apport d'ecdystérone en postmue (la femelle est en vitellogenèse primaire) induit la pré-

(1) On ne peut en effet assimiler la VSOH à l'ecdystérone (cf. plus loin). 
mue en $24 \mathrm{~h}$ mais ne permet pas le déclenchement de la vitellogenèse secondaire. Au contraire, la formation de l'épithélium folliculaire secondaire est inhibée (Blanchet-Tournier, 1980) ainsi que la synthèse de la vitellogénine (Blanchet et al., 1975 ; fig. 2, a).
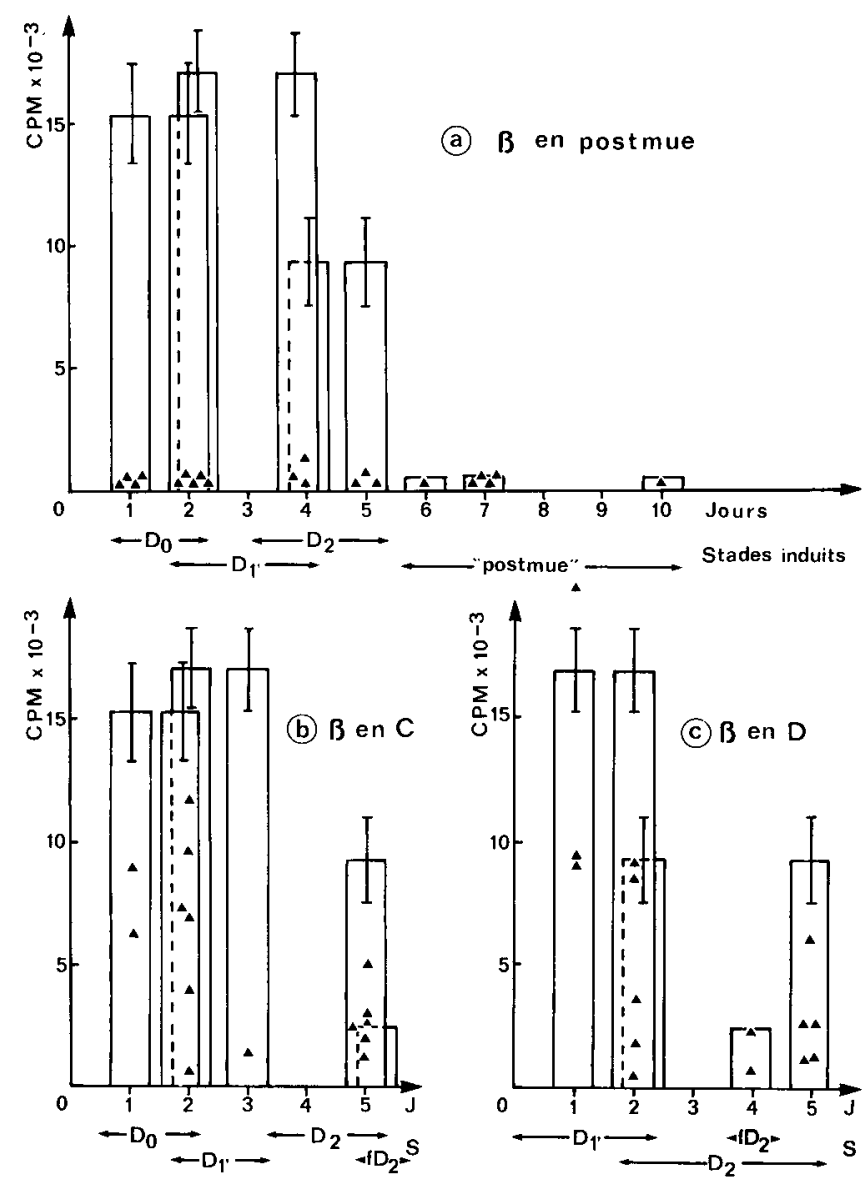

FIG. 2. - Effets de l'augmentation expérimentale du taux d'ecdystérone sur la synthèse de la vitellogénine chez des femelles $d^{\prime} O$. gammarellus en période de reproduction. Interprétation graphique des résultats publiés par Blanchet, Junéra et Meusy (1975).

a) Apport d'ecdystérone $(\beta)$ en postmue ; b) Apport d'ecdystérone durant la période $C$ de l'intermue ;

c) Apport d'ecdystérone durant la période $D$.

La radioactivité de la vitellogénine est exprimée en cpm/3 $\mu$ d'hémolymphe/6 $h$ d'incubation (a.s. de la leucine- ${ }^{3} \mathrm{H}: 50-60 \mathrm{Ci} / \mathrm{mM}$ ). Les triangles correspondent aux valeurs individuelles enregistrées chez les femelles expérimentales de 1 à 10 jours après l'apport d'ecdystérone ; leur stade de mue, induit par cette hormone, est indiqué sous l'échelle des abscisses. Les histogrammes représentent la synthèse de la vitellogénine enregistrée chez les femelles témoins aux mêmes stades de mue (moyennes $\pm \mathrm{ES}$; d'après Junéra, 1981).

"Postmue ": les femelles bien que possédant encore leur ancienne cuticule sont physiologiquement en postmue.

$D_{2}=$ fin $D_{2}$. 
Si l'augmentation expérimentale du taux d'ecdystérone survient alors que la femelle est en cours de vitellogenèse secondaire (période $\mathrm{C}$ ), la prémue, bien qu'induite rapidement (soit en $12 \mathrm{~h}$ ) dans 100 p. 100 des cas, n'est pas toujours suivie d'une exuviation précoce comme c'est le cas chez les mâles et les femelles en repos sexuel (Blanchet et Charniaux-Cotton, 1971). Les résultats sont d'ailleurs hétérogènes et sont probablement dus à la dose d'hormone de mue circulante. Nous les avons regroupés en 3 cas (fig. 3 ) :

- un déroulement très rapide de la prémue (6-7 jours au lieu de 12 jours chez les témoins) entraînant une exuviation anticipée de plus de 10 jours non suivie de ponte. Dans ce lot, bien que la synthèse de la vitellogénine ne soit pas stimulée (Blanchet et al., 1975 ; fig. 2, b), on observe une augmentation du taux de croissance des ovocytes qui peuvent atteindre $500 \mu \mathrm{m}$ lors de l'exuviation au lieu de $350 \mu \mathrm{m}$ chez des femelles témoins alors en fin de période $\mathrm{C}$;

- une prémue de durée quasi-normale (10 à 14 jours) ce qui permet, si l'avance de l'exuviation ne dépasse pas 7 jours, l'achèvement de la vitellogenèse secondaire et une ponte d'œufs viables bien que légèrement plus petits que la normale ;

- une prémue dont la durée peut dépasser 20 jours en raison d'un allongement des stades $D_{1 ' a}$ et $D_{2}$ ou du stade $D_{2}$ seul. Dans ce cas, on n'observe pas de stimulation de la vitellogenèse qui arrive à son terme dans un délai normal. L'exuviation est suivie de ponte et de développement des œufs.

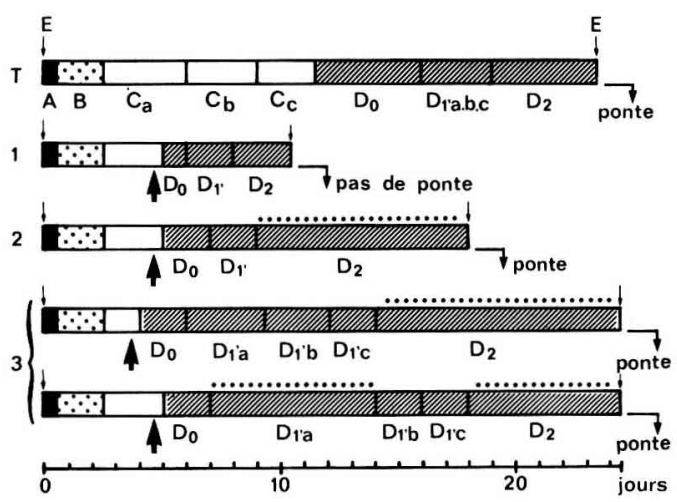

FIG. 3. - Influence de l'ovaire en vitellogenèse sur la durée de la prémue induite précocement par apport d'ecdystérone chez des femelles d'O. gammarellus au stade $C_{a}$. La prémue peut être très courte (cas 1), de durée normale (cas 2) ou très longue (cas 3). Dans les 2 derniers cas, où vitellogenèse et ponte se réalisent, un allongement significatif $(\ldots)$ du stade $D_{2}$ seul ou des stades $D_{1}$, et $D_{2}$ est observé.

T : Témoin ; E : exuviation : A, B. $C_{a} \ldots$ stades de mue et leurs durées respectives (se référer à l'échelle des jours); $\uparrow:$ apport d'ecdystérone.

En résumé, dans tous les cas exposés ci-dessus, qu'ils soient naturels ou expérimentaux, un déroulement plus rapide des phénomènes de mue est constaté en l'absence de vitellogenèse secondaire. A l'inverse, si la vitellogenèse secondaire se réalise jusqu'à son terme, on observe un allongement du cycle de mue ou 
même un ralentissement de certains stades de mue lorsque la prémue a été induite précocement.

Pour expliquer le déroulement plus rapide des phénomènes de mue en absence de reproduction, il n'est pas possible d'invoquer une augmentation du taux d'hormone de mue. En effet, la quantité d'ecdystéroïdes, dosés à un stade de mue donné, est la même chez les femelles en repos sexuel ou en reproduction (Blanchet et al., 1979). De même, l'hypothèse d'une augmentation de la sensibilité de l'épiderme à l'hormone de mue en hiver ne peut être retenue. Au contraire, il a été montré, chez le homard, que les organes-cibles sont moins sensibles à I'hormone de mue à faible température (Gilgan et Burns, 1977). La possibilité d'un antagonisme entre l'hormone de mue et un facteur inhibant la mue tel la MIH (Molt Inhibiting Hormone) ( ${ }^{2}$ ) peut être retenue. L'achèvement de la vitellogenèse secondaire s'accompagnant toujours d'une augmentation de la durée de l'intermue, nous postulons que l'ovaire en vitellogenèse secondaire est capable de stimuler la production de $\mathrm{MIH}$ par un centre nerveux qui reste à déterminer chez O. gammare/lus (cf. plus loin).

B. Etude du déroulement de la vitellogenèse secondaire en absence de mue. - Des interventions chirurgicales chez la femelle d'O. gammarellus en reproduction au niveau des organes $Y$ ou du protocérébron nous ont permis de bloquer le cycle de mue à différents stades. L'étude des phénomènes de vitellogenèse a été réalisée.

1. Microcautérisation des organes $Y$. - Les organes $Y$ d'O. gammarellus sont constitués de 2 massifs cellulaires céphaliques et symétriques (PI. I, 2).

a) Effets sur la mue : Les effets de la microcautérisation de ces organes sur le déroulement des phénomènes de mue chez la femelle en vitellogenèse sont les mêmes que ceux obtenus chez les mâles (Blanchet, 1974). Rappelons-les brièvement :

\begin{tabular}{|c|c|}
\hline Y-ectomie en B & $\begin{array}{l}\text { Achèvement de la postmue } \\
\text { Blocage du cycle de mue en } \mathrm{C}_{\mathrm{a}}\end{array}$ \\
\hline $\begin{array}{l}\text { Y-ectomie en } C \text {, } \\
D_{0} \text { et } D_{1}\end{array}$ & Blocage immédiat du cycle de mue \\
\hline Y-ectomie en $D_{2}$ & $\begin{array}{l}\text { Achèvement de la prémue } \\
\text { Exuviation } \\
\text { Réalisation de la postmue } \\
\text { Blocage au stade } C_{a} \text { du cycle post-opératoi }\end{array}$ \\
\hline
\end{tabular}

Les blocages observés indiquent que l'organe $Y$ est bien impliqué dans le contrôle des phénomènes préparatoires à l'exuviation et ceci quel que soit le sexe. Cette régulation se fait par l'intermédiaire des ecdystéroïdes ; en effet, l'organe $Y$ sécrète l'ecdysone (Chang et O'Connor, 1977, 1978) qui est rapidement métaboli-

(2) L'existence de cette hormone a été démontrée chez les Décapodes (voir revue Vernet, 1976). 
sée en ecdystérone (Lachaise et Feyereisen, 1976 ; Kuppert et al., 1978). Ces 2 hormones sont actives chez O. gammarellus (Blanchet, 1972).

b) Effets sur les phénomènes de vitellogenèse secondaire (Meusy et al., 1977 ; Blanchet-Tournier, 1980 ; et résultats non publiés) : Si la destruction des organes $Y$ est réalisée en postmue, il n'y a pas de démarrage de la vitellogenèse secondaire du fait probablement des perturbations observées lors de la mise en place du follicule secondaire. On note en effet l'absence de relations entre les ovocytes et les cellules folliculaires qui les entourent.

Si l'intervention survient en $C$ ou $D_{0}-D_{1}$, (la femelle est alors en cours de vitellogenèse secondaire), un arrêt de la croissance ovocytaire est observé (PI. I, 3). Après un délai de 20 à 30 jours, tandis que les ovocytes à croissance bloquée sont phagocytés par les cellules folliculaires qui les entourent, les ovocytes de la génération suivante restent en vitellogenèse primaire. Enfin, si la femelle est opérée en $D_{2}$, la vitellogenèse proche de son terme s'achève mais il n'y aura pas de nouvelle vitellogenèse secondaire lors du cycle post-opératoire.

Ainsi, dans cette série expérimentale, le blocage des phénomènes de mue s'accompagne d'un arrêt de la vitellogenèse voire même d'une absence complète de vitellogenèse si l'on intervient avant qu'elle ne soit engagée.

Des résultats similaires ont été obtenus chez deux Peracarides qui offrent ce même synchronisme mue-vitellogenèse : /dotea balthica (Reidenbach, 1971), Porcellio dilatatus (Besse et Maissiat, 1971). L'organe $Y$ apparaît donc bien impliqué dans le processus de reproduction comme il l'est lors de la croissance.

Chez O. gammarellus, la destruction des organes $Y$ en postmue empêche également le déclenchement de la synthèse de la vitellogénine (fig. 4). En raison

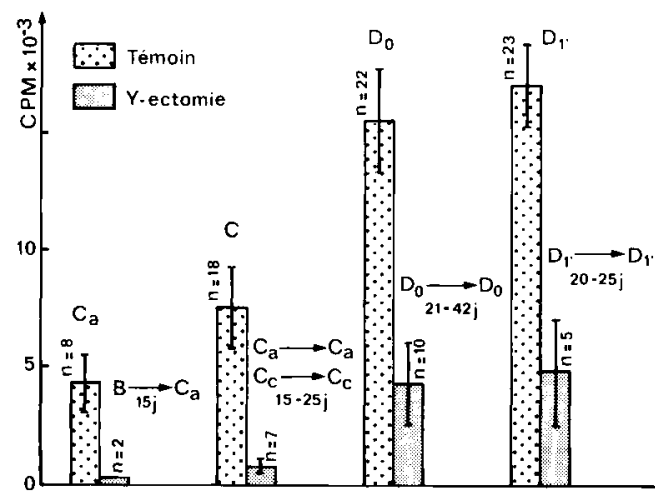

FIG. 4. - Effets de la destruction des organes $Y$ sur la synthèse de la vitellogénine chez des femelles d'O. gammare/lus. Interprétation graphique des résultats publiés par Meusy, Blanchet, Junéra (1977) et d'expériences complémentaires réalisées par ces mêmes auteurs.

La radioactivité de la vitellogénine est exprimée en $\mathrm{cpm} / 3 \mu \mathrm{l}$ d'hémolymphe/6 heures d'incubation (a.s. de la leucine ${ }^{3} \mathrm{H}: 55-57 \mathrm{Ci} / \mathrm{mM}$ ).

La destructıon des organes $Y$, réalisée en $B, C_{a}, C_{c}, D_{0}$, et $D_{1}$, entraîne un blocage du cycle de mue respectivement en $C_{a}, C_{c}, D_{0}$ et $D_{1}$. L La synthèse de la vitellogénine, étudiée de 15 à 42 jours après l'opération (moyennes \pm ES) est comparée à celle de femelles témoins aux mêmes stades de mue (moyennes \pm ES; d'après Junéra, 1981). La différence est hautement significative. 
des anomalies observées au niveau du follicule, nous avons émis I'hypothèse que le tissu folliculaire secondaire était alors incapable de sécréter la VSOH d'où l'absence d'activité de synthèse du tissu adipeux. Pratiquée au cours de la vitellogenèse secondaire, la cautérisation de l'organe $Y$ provoque une forte diminution de la synthèse de la vitellogénine (fig. 4) et un arrêt de la croissance ovocytaire (cf. plus haut). La vitellogénine étant présente dans I'hémolymphe, la participation de l'organe $Y$ au contrôle de son entrée dans les ovocytes ne fait aucun doute. De plus, bien que les variations du taux des ecdystéroïdes ne soient pas parallèles aux variations de la synthèse de la vitellogénine (fig. 1, b, d, e) nous pensons pouvoir affirmer que les ecdystéroïdes (ecdysone ou ecdystérone (?)) sont nécessaires au maintien de la production de vitellogénine en stimulant le tissu adipeux directement ou par l'intermédiaire de la VSOH. Bien qu'il existe des ecdystéroïdes ovariens chez $O$. gammarellus, l'ovaire et l'organe $Y$ étant à la fois nécessaires pour une synthèse normale de vitellogénine, la VSOH ne peut être assimilée à l'ecdysone comme cela a été suggéré chez le moustique Aedes aegypti (Hagedorn et al., 1975). Enfin, la chute du taux des ecdystéroïdes observée en fin d'intermue (fig. 1, d, e) pourrait provoquer l'abaissement de la sécrétion de vitellogénine.

2. Destruction de la région antéro-médiane du protocérébron. - Le protocérébron est formé de 2 masses globuleuses symétriques, séparées par un sillon médian étroit et profond et auxquelles sont rattachés les lobes optiques. Plusieurs types de cellules neurosécrétrices, occupant la périphérie du protocérébron sont détruites lors de la microcautérisation.

a) Effets sur la mue (Blanchet-Tournier, 1979): Comme dans le cas de I'Y-ectomie, l'état de reproduction de la femelle n'influence pas les effets sur la mue constatés en absence de cette région cérébrale. Nous les résumons comme suit : (P- : destruction de la partie antéro-médiane du protocérébron) :

$$
\begin{aligned}
& P \text { - en } B \text { et } C \quad \text { Période } C \text { ralentie } \\
& \text { Blocage du cycle en fin de } C \text { ou début } D_{0} \\
& P \text { - en } D_{0} \text { let par- } \\
& \text { fois en } D_{1^{\prime} a} \text { ) Blocage immédiat du cycle de mue } \\
& P \text { - en } D_{1 ' b-c} \text { et } D_{2} \quad \text { Fin de prémue ralentie } \\
& \text { Exuviation } \\
& \text { Intermue (postmue + période C) post-opératoire } \\
& \text { ralentie } \\
& \text { Blocage en } D_{0} \text {. }
\end{aligned}
$$

Le ralentissement des phénomènes de mue, voire même l'impossibilité d'une apolyse générale chez les animaux " décérébrés » ne nous permettent pas d'appliquer à $O$. gammarel/us l'hypothèse selon laquelle 2 hormones antagonistes protocérébrales : MIH et MAH (Molt Accelerating Hormone) seraient sécrétées alternativement au cours du cycle de mue comme c'est le cas chez les mâles et les femelles de Porcellio dilatatus (Martin et al., 1979. 1980). L'existence d'un seul facteur protocérébral qui stimulerait directement ou indirectement la mue et dont 
l'action s'exercerait notamment en $D_{0}$ peut expliquer l'ensemble des résultats obtenus. Trois hypothèses sont actuellement à envisager :

- Le facteur protocérébral serait une hormone du type "YSH " (= Y-organ Stimulating Hormone) comme l'a suggéré Charmantier (1980) chez le Sphérome, favorisant la synthèse et/ou la libération d'ecdysone par l'organe $Y$. Cette hormone serait alors à rapprocher de la " PTTH = Prothoracotropic Hormone " isolée chez les Insectes (Nagasawa et al., 1979) et qui induit la glande prothoracique à produire de l'ecdysone (Bollenbacher et al., 1979). Le taux des ecdystéroïdes chez des mâles d'O. gammarellus " décérébrés " et bloqués en $D_{0}$, étant supérieur à celui des témoins au même stade, il ne semble pas possible de retenir cette première hypothèse.

- Le facteur protocérébral serait nécessaire à la réponse de l'épiderme à I'hormone de mue. Signalons dès à présent qu'une augmentation expérimentale du taux d'ecdystérone chez des animaux « décérébrés » et bloqués en $D_{0}$, induit immédiatement la prémue et conduit à l'exuviation (résultats non publiés).

Chez les Vertébrés, il est connu que certains stéroïdes (tel l'œestradiol) peuvent augmenter le nombre de leurs récepteurs au niveau des organes-cibles (Baulieu, 1978). Ce même effet peut être provoqué par les neuropeptides. Si de tels schémas sont applicables aux Invertébrés, ils apporteraient des arguments en faveur de cette deuxième hypothèse. Le nombre de récepteurs à l'hormone de mue au niveau de l'épiderme serait sous l'influence du protocérébron, ce qui expliquerait le ralentissement des phénomènes de mue en son absence. L'ecdystérone, en excès dans l'hémolymphe, augmentant ses propres récepteurs, l'épiderme pourrait répondre à l'hormone même en absence du protocérébron.

- Le facteur protocérébral inhiberait la production de MIH par un autre centre nerveux. L'action antagoniste entre la MIH et l'hormone de mue au niveau épidermique ayant été démontrée (Freeman et Bartell, 1976 ; Freeman et Costlow, 1979), le ralentissement, voire le blocage des phénomènes de mue après " décérébration "), serait lié chez $O$. gammarellus à la quantité de MIH produite. Une augmentation expérimentale du taux d'ecdystérone pourrait alors permettre la reprise des processus de mue, comme nous l'avons observé.

Les données actuellement acquises chez 0 . gammarellus ne permettent pas de trancher entre les $2^{\mathrm{e}}$ et $3^{\mathrm{e}}$ hypothèses.

b) Effets de la "décérébration" sur les phénomènes de vitellogenèse (Blanchet-Tournier, 1980 ; Blanchet-Tournier et al., 1980 et résultats non publiés): L'étude des ovaires a été réalisée chez des femelles "décérébrées 》 avant la reprise de la vitellogenèse secondaire ou au cours de celle-ci.

Toutes les femelles opérées en fin de cycle de mue $\left(D_{2}\right)$ ont exuvié mais $3 / 4$ d'entre elles ne sont pas entrées en vitellogenèse secondaire dans un délai normal. II en est de même pour les femelles opérées en $B$ ou début $C_{a}$. Des observations histologiques à différents délais après l'intervention indiquent que la folliculogenèse est très ralentie : elle s'étend en effet sur 10-20 jours au lieu de 2-3 jours chez les femelles témoins. Un retard important de la croissance ovocytaire est observé dans ces deux lots de femelles ainsi qu'une absence de synthèse de vitellogénine pour la pluplart d'entre elles. Lorsque la folliculogenèse est achevée, la 
vitellogenèse secondaire peut se réaliser jusqu'à son terme. En effet, des femelles opérées en début $C_{a}$ et étudiées 35 jours plus tard (leur cycle de mue est bloqué en fin $C$ ou début $D_{0}$ ) sont en fin de vitellogenèse secondaire. La taille de leurs ovocytes est d'environ $700 \times 650 \mu \mathrm{m}$; la vésicule germinative a terminé sa migration mais il $n^{\prime} y$ a pas de rupture de la membrane nucléaire. Le cytoplasme contient du matériel APS ${ }^{+}$en plaquettes et des lipides qui forment parfois d'énormes plages. Les macrovillosités de la membrane ovocytaire peuvent être rétractées laissant un espace entre l'ovocyte et les cellules folliculaires qui sont très aplaties.

Les femelles opérées en cours de vitellogenèse secondaire, soit en $C$ ou $D_{0}$, et bloquées à ce dernier stade, poursuivent leur croissance ovocytaire dans un délai quasi-normal. Ainsi, chez des femelles cautérisées en $D_{0}$, les ovocytes ont, 13 jours plus tard, une taille de $650 \times 520 \mu \mathrm{m}$. La vésicule germinative a migré en périphérie et le cytoplasme est envahi, comme chez les témoins en vitellogenèse avancée, de plaquettes vitellines et de globules lipidiques (PI. I, 4). La synthèse de la vitellogénine se poursuit chez ces femelles pendant un certain temps ( $\geq 18$ jours) puis diminue fortement (observation faite 35 jours après l'opération). L'ovaire est alors au terme de la vitellogenèse, les ovocytes pouvant présenter un début de résorption.

Ainsi, chez $O$. gammarellus, la destruction du protocérébron, en début ou en fin d'intermue, entraîne un retard important du démarrage de la vitellogenèse secondaire dû probablement au ralentissement de la formation des follicules secondaires, ceux-ci étant alors inaptes à sécréter la VSOH.

Des résultats obtenus chez les Isopodes par Besse (1968), Besse et al. (1969) et Mocquard et al. (1971) indiquent au contraire que la région médiane du protocérébron exerce une action inhibitrice sur la vitellogenèse. Reidenbach (1971) précise en outre que ce sont « les éléments neurosécréteurs homologues de l'organe de Hanström et localisés dans la région intercérébrale antérieure du protocérébron " qui sont seuls responsables de la fonction gonado-inhibitrice chez I'Isopode Idotea balthica. Les résultats différents exposés ci-dessus ne permettent pas une telle homologation chez $O$. gammarellus.

Lorsque la vitellogenèse secondaire est engagée chez $O$. gammarellus, son achèvement est possible en l'absence du protocérébron et donc en l'absence de prémue (le déroulement de l'intermue est en effet bloqué en fin de période $\mathrm{C}$ ou en $D_{0}$ ) ; la synthèse de la vitellogénine se poursuit normalement pendant un certain temps. Ce résultat n'est pas incompatible avec celui obtenu chez les femelles $\mathrm{d}^{\prime} O$. gammarellus $\mathrm{Y}$-ectomisées pour lesquelles le blocage des phénomènes de mue s'accompagne d'un blocage de la croissance ovocytaire et d'une forte diminution de la synthèse de la vitellogénine. En effet, les individus "décérébrés " bloqués en $D_{0}$ présentent une concentration en ecdystéroïdes supérieure à la normale. Ce taux hormonal permettrait la poursuite de la synthèse de la vitellogénine et de la croissance ovocytaire. A l'inverse, la concentration faible $\left(^{3}\right) d^{\prime}$ 'ecdystérö̈des, après $\mathrm{Y}$-ectomie, provoquerait la diminution de la synthèse de la vitellogénine et l'arrêt de l'entrée de matériel vitellin dans les ovocytes.

(3) En l'absence des organes $Y$, de petites quantités d'ecdystéroïdes persistent chez le Crabe Eriocheir sinensis qui est bloqué en $\mathrm{C}_{4}$ (de Leersnyder et al., 1980) ainsi que chez le Crabe Pachygrapsus marmoratus (Charmantier-Daures, 1980). 
Mécanismes hormonaux assurant la coordination entre la mue et la vitellogenese chez 0 . gammarellus.

L'ensemble de ces résultats apporte de nouvelles données quant au synchronisme mue-vitellogenèse chez 0 . gammarellus.

Le déclenchement de la vitellogenèse secondaire apparaît très lié à l'état physiologique de la femelle en fin de postmue, toute intervention (augmentation du taux d'ecdystérone, destruction des organes $Y$ ou du protocérébron) inhibant momentanément ou définitivement ce processus. Quel est l'environnement hormonal qui caractérise normalement cette période ? Le taux d'ecdystéroïdes dosés dans l'hémolymphe des femelles ainsi que dans les extraits totaux est alors très faible. Cette quantité d'hormones de mue, due probablement à une activité de synthèse faible de l'organe $Y$ est cependant nécessaire. En effet, l'Y-ectomie empêche un déroulement normal du cycle de mue au-delà du stade $C_{a}$ et inhibe le déclenchement des phénomènes de vitellogenèse secondaire (synthèse de vitellogénine et croissance ovocytaire). Cependant ces ecdystéroïdes ne représentent pas le seul stimulus ; en effet, à une même dose, ces hormones ne suffisent pas à déclencher la vitellogenèse secondaire chez des femelles d'O. gammarellus en repos sexuel hivernal. Le système nerveux central intégrant les facteurs externes favorables (photophase et température) est certainement impliqué dans le contrôle du démarrage de la vitellogenèse secondaire. Le protocérébron (seul centre nerveux ayant fait l'objet d'une étude chez $O$. gammarellus) semble servir de relais à l'information reçue au niveau des récepteurs sensoriels. En effet, la destruction des cellules neurosécrétrices protocérébrales entraîne un retard considérable du démarrage de la vitellogenèse secondaire.

Les " signaux » émis par le protocérébron et l'organe $Y$ seraient perçus par la gonade comme en témoignent les anomalies observées en l'absence de ces organes lors de la formation des follicules secondaires. Chez la femelle normale, l'activité endocrine de l'ovaire, c'est-à-dire la synthèse de la VSOH, ne pourrait se déclencher en $C_{a}$ que si les cellules folliculaires ont achevé leur mise en place et que si des relations sont établies entre les ovocytes et leur enveloppe. La VSOH stimulerait la synthèse de vitellogénine par le tissu adipeux. Cette hétéroprotéine circulant dans l'hémolymphe est captée par les ovocytes qui amorcent ainsi leur grande croissance.

Lorsque la vitellogenèse secondaire est déclenchée, quels sont les mécanismes qui assurent sa poursuite tout au cours de l'intermue ?

La destruction des organes $Y$ entraîne un arrêt de la vitellogenèse secondaire concomitant de celui de la mue. Ces résultats indiquent la nécessité de la présence des ecdystéroïdes pour permettre un déroulement normal de la vitellogenèse secondaire. Chez les femelles privées de la région médiane du protocérébron, le blocage de la mue n'est pas accompagné d'un blocage de la vitellogenèse, celle-ci se poursuivant jusqu'à son terme, probablement grâce au taux important d'ecdystéroïdes. Ces faits confirment l'intervention des hormones de mue dans le déroulement de la vitellogenèse.

Notons en outre que la croissance ovocytaire n'est pas directement en rela- 
tion avec la quantité de vitellogénine produite. C'est le cas de la femelle normale (fig. 1, a, b) et des femelles $Y$-ectomisées. Chez ces dernières, les ovocytes subissent un arrêt de leur croissance tandis que la synthèse de la vitellogénine se poursuit, quoique faiblement. La régulation des phénomènes de vitellogenèse par les ecdystéroïdes serait donc assurée à deux niveaux au moins : - au niveau de la capture de la vitellogénine par les ovocytes ; - au niveau des cellules folliculaires qui produisent la VSOH. Cependant, I'intervention directe des ecdystéroïdes sur l'activité de synthèse du tissu adipeux n'est pas à exclure.

La reproduction modifie la durée de l'intermue provoquant soit un allongement du cycle de mue soit une suspension temporaire des phénomènes de mue induits précocément par injection d'ecdystérone. Ces observations suggèrent l'existence d'un facteur antagoniste de l'hormone de mue tel la MIH dont la synthèse serait stimulée par l'ovaire en vitellogenèse secondaire.

En résumé, le synchronisme étroit observé chez la femelle d' $O$. gammarellus entre la mue et la vitellogenèse serait assuré par des concentrations variables d'hormone de mue et de MIH ; la première étant nécessaire à la fois à la croissance et à la vitellogenèse, la seconde assurant un contrôle inhibiteur des phénomènes de mue. La synthèse de MIH serait également contrôlée : l'ovaire en vitellogenèse (par l'intermédiaire de la VSOH (?)) stimulerait cette production tandis que le protocérébron l'inhiberait.

La fin de la vitellogenèse secondaire est marquée par une diminution importante de l'activité de synthèse du tissu adipeux. Les ovocytes, dissociés de leur enveloppe folliculaire, cessent toute croissance. L'épiderme achève alors la sécrétion des couches pré-exuviales de la cuticule, c'est l'étape $D_{2}$ caractérisée notamment par un pic d'ecdystéroïdes suivi d'une chute brutale du taux de ces hormones.

L'arrêt des phénomènes de vitellogenèse peut également être observé même si la femelle n'a pas subi cette variation hormonale importante qui marque la fin de l'intermue et de la vitellogenèse. C'est le cas de ces femelles qui, privées de la région médiane du protocérébron et bloquées au stade $D_{0}$ de l'intermue, présentent cependant toutes les caractéristiques d'une fin de vitellogenèse : synthèse de vitellogénine ralentie, taille ovocytaire maximale et dissociation ovocyte-épithélium folliculaire.

Ces résultats suggèrent la possibilité d'un contrôle de l'arrêt des phénomènes de vitellogenèse par l'ovaire lui-même qui, du fait de la présence d'ovocytes mûrs ou (et) de leur séparation de leur enveloppe folliculaire cesserait de produire la $\mathrm{VSOH}$ et de capter la vitellogénine.

\section{Conclusion.}

Le schéma (fig. 5) que nous proposons ici récapitule les données actuellement acquises chez 0 . gammarellus concernant le contrôle endocrine de la vitellogenèse et de la mue.

Le déclenchement de la vitellogenèse secondaire est lié à une folliculogenèse normale qui se réalise en postmue sous le contrôle du protocérébron et en pré- 
sence d'un taux faible d'ecdystéroïdes. L'ovaire élabore alors la VSOH qui stimule le tissu adipeux à produire la vitellogénine.

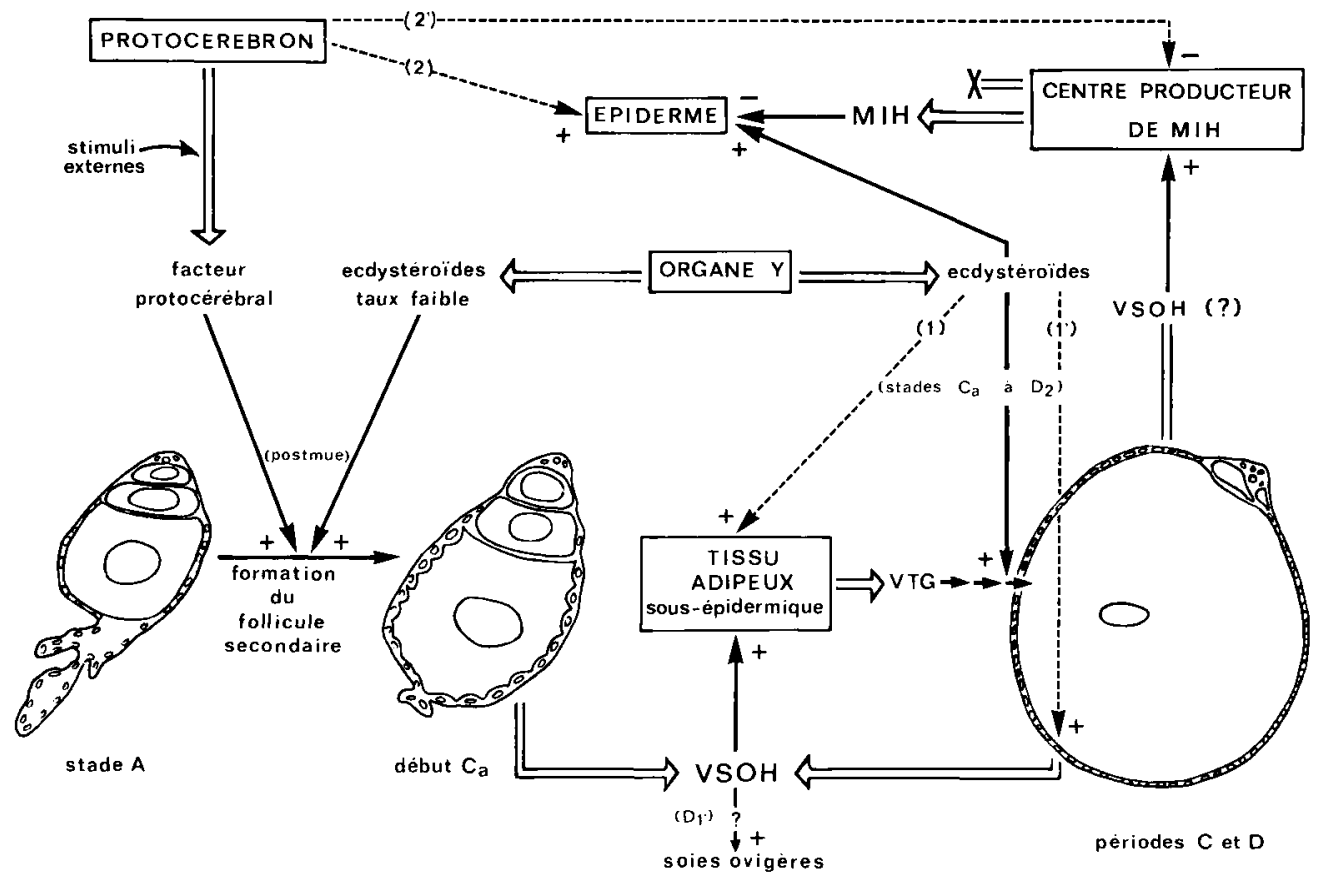

FIG. 5. - Représentation schématique des interactions entre les organes contrôlant la mue et l'ovaire en vitellogenèse chez la femelle pubère d'O. gammarellus. $\Longrightarrow$ production : action stimulatrice $(+)$ ou inhibitrice $(-) ;-\rightarrow \rightarrow$ action hypothétique stimulatrice $(+)$ ou inhibitrice $(-)$. VSOH, hormone ovarienne stimulant la synthèse de vitellogénine ; VTG, vitellogénine ; $\mathrm{MIH}$, hormone inhibitrice de la mue. Stades $\mathrm{A}, \mathrm{C}_{\mathrm{a}} \ldots$ stades de l'intermue.

Le déroulement de la vitellogenèse secondaire, durant les périodes $C$ et $D$ du cycle de mue, nécessite la synthèse continue de vitellogénine et la capture de cette hétéroprotéine circulante par les ovocytes. Durant ces périodes, l'activité de synthèse du tissu adipeux est stimulée par la VSOH qui agirait en synergie avec I'hormone de mue (voie 1) ou bien par la VSOH seule, les ecdystéroïdes stimulant la production de cette hormone au niveau ovarien (voie 1 '). L'entrée de la vitellogénine dans les ovocytes est sous le contrôle de l'organe $Y$ par l'intermédiaire des ecdystéroïdes.

L'ovaire en cours de vitellogenèse secondaire stimulerait (via la VSOH ?) la production de $\mathrm{MIH}$; cette hormone, antagoniste de I'hormone de mue au niveau épidermique, entraînant un ralentissement des phénomènes préparatoires à l'exuviation.

Enfin, indépendamment de leur action sur la vitellogenèse, l'organe $Y$ et le protocérébron contrôlent les phénomènes de mue. Deux voies de régulation sont possibles pour le centre nerveux protocérébral : une activation directe de l'épiderme (2) ou bien une inhibition du centre producteur de $\mathrm{MIH} \mathrm{(2').}$ 
Remerciements. - Nous remercions Madame le Professeur $\mathrm{H}$. Charniaux-Cotton pour la lecture critique de ce manuscrit et Mademoiselle Martin pour sa collaboration technique.

\section{Références}

BALESDENT M. L., 1964. Recherches sur la sexualité et le déterminisme des caractères sexuels $d^{\prime}$ Asellus aquaticus Linné (Crustacé /sopode). Th. Doct. Etat, Univ. Nancy.

BAULIEU E. E., 1978. Hormones. Aspects fondamentaux et physio-pathologiques. Hermann.

BESSE G., 1968. Contribution à l'étude du contrôle neurohumoral de la maturation ovarienne et de la mue parturielle chez l'Oniscoïde Porcellio dilatatus Brandt. C. R. Acad. Sci. Paris, sér. $D, 266,917-919$.

BESSE G., 1976. Contribution à l'étude expérimentale de la physiologie sexuelle femelle chez les Crustacés /sopodes terrestres. Th. Doct. Etat, Poitiers, CNRS AO 13 017, 261 pp.

BESSE G., JUCHAULT P., LEGRAND J.J., MOCQUARD J. P., 1969. Contribution à l'étude de la physiologie sexuelle femelle de Ligia oceanica $L$. (Crustacé Oniscoöde). Différenciation des oostégites et contrôle neurohumoral de la maturation ovarienne. C. R. Acad. Sci. Paris, sér. $D, 269,733-736$.

BESSE G., MAISSIAT J., 1971. Action de la glande de mue sur la vitellogenèse du Crustacé Isopode Porcellio dilatatus (Brandt). C. R. Acad. Sci. Paris, sér. D, 273, 1975-1978.

BLANCHET M.-F., 1972. Effets sur la mue et sur la vitellogenèse de la $\beta$-ecdysone introduite aux étapes $A$ et $D_{2}$ du cycle d'intermue chez Orchestia gammarella Pallas (Crustacé Amphipode). Comparaison avec les effets de la $\beta$ - et de l' $\alpha$-ecdysone aux autres étapes de l'intermue. C. R. Acad. Sci. Paris, sér. D, 274, 3015-3018.

BLANCHET M.-F., 1974. Etude du contrôle hormonal du cycle d'intermue et de l'exuviation chez Orchestia gammarella par microcautérisation des organes $Y$ suivie d'introduction d'ecdystérone. C. R. Acad. Sci. Paris, sér. D, 278, 509-512.

BLANCHET-TOURNIER M.-F., 1979. Etude des effets de la destruction du protocérébron sur les phénomènes de mue chez le Crustacé Amphipode Orchestia gammarellus. C. R. Acad. Sci. Paris, sér. D, 289, 335-337.

BLANCHET-TOURNIER M.-F., 1980. Etude du contrôle endocrine du démarrage de la vitellogenèse secondaire chez le Crustacé Amphipode Orchestia gammarellus (Pallas). C. R. Acad. Sci. Paris, sér. D, 291, 969-972.

BLANCHET M.-F., CHARNIAUX-COTTON H., 1971. Contrôle du déclenchement et de la durée de la période $D$ du cycle d'intermue par l'ecdystérone, chez le Crustacé Amphipode Orchestia gammarella (Pallas); interaction avec la vitellogenèse. C. R. Acad. Sci. Paris, sér. D, 272, 307-310.

BLANCHET M.-F., JUNERA H., MEUSY J.-J., 1975. Mue et vitellogenèse chez Orchestia gammarella Pallas, Crustacé Amphipode : étude de la synthèse de la fraction protéique femelle après introduction d'ecdystérone. Experientia, 31, 865-867.

BLANCHET M.-F., PORCHERON P., DRAY F., 1979. Variations du taux des ecdystéroïdes au cours des cycles de mue et de vitellogenèse chez le Crustacé Amphipode, Orchestia gammarellus. Int. J. Invertebr. Reprod., 1, 133-139.

BLANCHET-TOURNIER M.-F., MEUSY J.-J., JUNERA H., 1980. Mue et vitellogenèse chez le Crustacé Amphipode Orchestia gammarellus (Pallas). Etude des effets de la destruction de la région antéro-médiane du protocérébron sur la synthèse de la vitellogénine. C. R. Acad. Sci., Paris, sér. D, 291, 829-832.

BOLLENBACHER W. E., AGUI N., GRANGER N. A., GILBERT L.I., 1979. In vitro activation of Insect prothoracic glands by the prothoracicotropic hormone. Proc. nat. Acad. Sci., USA, 76, 5148-5152.

BRODZICKI S., 1963. Localization of lipids acid $\alpha$-ketolic steroids in the ovary of Crustacea. Folia histochem. cytochem., 1, 259-268.

CHAIX J.-C., de REGGI M., 1979. Variations des ecdystéroüdes durant la vitellogenèse et l'embryogenèse du Crabe Oxyrhynque, Acanthonyx lunulatus (Risso). Journ. Soc. zool. Fr., Poitiers. 
CHANG E. S., O'CONNOR J. D., 1977. Secretion of $\alpha$-ecdysone by crab Y-organs in vitro. Proc. nat. Acad. Sci., USA, 74, 615-618.

CHANG E. S., O'CONNOR J. J., 1978. In vitro secretion and hydroxylation of $\alpha$-Ecdysone as a function of the Crustacean molt cycle. Gen. comp. Endocrinol., 36, 151-160.

CHARMANTIER G., 1980. Contrôle endocrine et neuroendocrine de la mue de puberté chez les mâles de Sphaeroma serratum (Fabricius, 1787) (Crustacea, Isopoda, Flabellifera). Gen. comp. Endocrinol., 41, 349-364.

CHARMANTIER-DAURES M., 1980. La mue et la régénération chez Pachygrapsus marmoratus (Fabricius, 1787), (Crustacé, Décapode, Brachyoure), Interactions, contróles endocrine et neuroendocrine. Th. Doct. Etat, Montpellier, 233 pp.

CHARNIAUX-COTTON $H_{\text {., }}$ 1957. Croissance, régénération et déterminisme endocrinien des caractères sexuels d'Orchestia gammarella Pallas (Crustacé Amphipode). Ann. Sci. nat. zool., 11e sér., 413-559.

CHARNIAUX-COTTON H., 1973. Description et contrôle de l'ovogenèse chez les Crustacés Supérieurs. Ann. Biol. anim. Bioch. Biophys., 13 (hors série), 21-30.

CHARNIAUX-COTTON H., 1974. Données nouvelles concernant la vitellogenèse des Crustacés Malacostracés obtenues chez l'Amphipode Orchestia gammarellus (Pallas) : folliculogenèse à partir d'un tissu permanent; action du busulfan; action inhibitrice de l'hormone juvénile. C. R. Acad. Sci. Paris, sér. D, 279, 563-566.

CHARNIAUX-COTTON H., 1975. L'ovogenèse et sa régulation chez les Crustacés Supérieurs. Ann. Biol. anim. Bioch. Biophys., 15, 715-724.

CHARNIAUX-COTTON H., 1978. L'ovogenèse, la vitellogénine et leur contrôle chez le Crustacé Amphipode Orchestia gammarellus (Pallas). Comparaison avec d'autres Malacostracés. Arch. Zool. exp. gén., 119, 365-397.

CHARNIAUX-COTTON H., 1980. Experimental studies of reproduction in Malacostraca Crustaceans. Description of vitellogenesis and of its endocrine control. W. H. CLARK Jr, T. S. ADAMS, Advances in Invertebrate Reproduction, Development in Endocrinology, 11, 177-186. Elsevier North Holland, Inc.

CHARNIAUX-COTTON H., TOUIR A., 1973. Contrôle de la prévitellogenèse et de la vitellogenèse chez la Crevette hermaphrodite Lysmata seticaudata Risso. C. R. Acad. Sci. Paris, sér. $D, 276,2717-2720$.

CHEUNG T. S., 1966. The interrelations among three hormonal-controlled characters in the adult female shore Crab. Carcinus maneas (L.). Biol. Bull., 133, 59-66.

CROISILLE Y., JUNÉRA H., 1980. Recherche du lieu de synthèse de la vitellogénine chez le Crustacé Amphipode Orchestia gammarella (Pallas). II. Démonstration, à l'aide d'anticorps spécifiques, de la présence de vitellogénine dans le tissu adipeux sous-épidermique des femelles en vitellogenèse secondaire. C. R. Acad. Sci. Paris, sér. D, 290, 1487-1490.

DEMEUSY N., 1963. Rapports entre mue et vitellogenèse chez le Crabe Carcinus maenas L. Proceed. 16th Congr. int. Zool., Washington, 118.

DESCOUTURELLES G., 1978. Influence de l'ablation des pédoncules oculaires sur la longévité, l'évolution ovarienne et la durée du cycle d'intermue chez la Crevette d'eau douce Atyaephyra desmaresti Millet 1831. Etude des facteurs température, saison et sexualité. Arch. Zool. exp. gen., 119, 433-445.

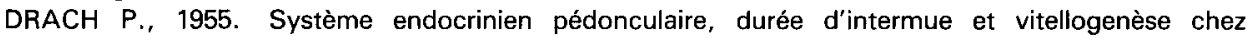
Leander serratus (Pennant), Crustacé Décapode. C. R. Soc. Biol., 149, 2079-2083.

FREEMAN J. A., BARTELL C. K., 1976. Some effects of the molt-inhibiting hormone and 20-hydroxyecdysone upon molting in the Grass sphrimp, Palaemonetes pugio. Gen. comp. Endocrinol., 28, 131-142.

FREEMAN J. A., COSTLOW J. D., 1979. Hormonal control of apolysis in Barnacle mantle tissue epidermis, in vitro. J. exp. Zool., 210, 333-346.

GILGAN M. W., BURNS B. G., 1977. On the reduced sensitivity of the adult male lobster (Homarus americanus) to ecdysterone at reduced temperature. Comp. Biochem. Physiol., 58 A, 33-36.

HAGEDORN H. H., O'CONNOR J. D., FUCHS M. S., SAGE G., SCHLAEGER D. A., BOHM M. K., 1975. The ovary as a source of $\alpha$-ecdysone in an adult mosquito. Proc. nat. Acad. Sci., U.S.A., 72, 3235-3259. 
JUNERA H., 1981. La vitellogénine chez le Crustacé Amphipode Orchestia gammarellus (Pallas). Propriétés physico-chimiques, transformation en lipovitellines, localisation et contrôle endocrine de sa synthèse. Th. Doct. Etat, Univ. Paris VI, 259 pp.

JUNÉRA H., ZERBIB C., MARTIN M., MEUSY J.-J., 1977. Evidence for control of vitellogenin synthesis by an ovarian hormone in Orchestia gammarella (Pallas), Crustacea, Amphipoda. Gen. comp. Endocrinol., 31, 457-462.

JUNÉRA H., CROISILLE Y., 1980. Recherche du lieu de synthèse de la vitellogénine chez le Crustacé Amphipode Orchestia gammarella (Pallas). Mise en évidence d'une activation de la synthèse protéique dans le tissu adipeux sous-épidermique en liaison avec la production de vitellogénine. C. R. Acad. Sci. Paris, sér. D, 290, 703-706.

KUPPERT P., BÜCHLER M., SPINDLER K. D., 1978. Distribution and transport of molting hormones in the Crayfish, Orconectes limosus. Z. Naturforsch., 33 C, 437-441.

LACHAISE F., 1981. Ecdystérö̈des et reproduction chez le Crabe Carcinus maenas (L.). Th. Doct. Etat. Univ. Paris VI.

LACHAISE F., FEYEREISEN R., 1976. Métabolisme de l'ecdysone par divers organes de Carcinus maenas (L) incubés in vitro. C. R. Acad. Sci. Paris, sér. D, 283, 1445-1448.

LACHAISE F., HOFFMANN J. A., 1977. Ecdysone et développement ovarien chez un Décapode, Carcinus maenas. C. R. Acad. Sci., Paris, sér. D, 285, 701-704.

LEERSNYDER M. de, DHAINAUT A., PORCHERON P., 1980. La vitellogenèse chez le Crabe Eriocheir sinensis. Bull. Soc. Zool. Fr., 105, 413-419.

LEGRAND J.-J., 1958. Induction de la maturité ovarienne et de la mue parturiale par la fécondation chez I'Oniscoïde Porcellio dilatatus. C. R. Acad. Sci. Paris, sér. D, 247, 754-757.

MARTIN G., MOCQUARD J. P., BESSE G., 1979. Contrôle neurohumoral de la mue chez les femelles de l'Oniscoïde Porcellio dilatatus Brandt. Bull. Soc. Sci. nat. Tunisie, 14, 47-58.

MARTIN G., BESSE G., MOCQUARD J. P., 1980. Contrôle neurohumoral du cycle de mue chez les mâle de l'Oniscoïde Porcellio dilatatus Brandt. Bull. Soc. Zool. Fr., 105, 73-81.

MATHIEU-CAPDEROU C., 1980. Relation entre la maturation ovocytaire et l'exuviation chez le Crustacé Amphipode Orchestia gammarellus (Pallas). C. R. Acad. Sci., Paris, sér. D, 290. 1495-1498.

MEUSY J.-J., 1980. Vitellogenin, the extraovarian precursor of the protein yolk in Crustacea : a review. Reprad. Nutr. Dévelop., 20, 1-21.

MEUSY J.-J., JUNERA H., CROISILLE Y., 1974. Données sur la synthèse de la fraction protéique femelle chez Orchestia gammarella Pallas (Crustacé Amphipode), au cours de l'intermue et chez les femelles en repos sexuel. C. R. Acad. Sci., Paris, sér. D, 279, 587-590.

MEUSY J.-J., BLANCHET M. F., JUNÉRA H., 1977. Mue et vitellogenèse chez le Crustacé Amphipode Orchestia gammarella Pallas. II. Etude de la synthèse de la vitellogénine (« Fraction protéique femelle " de l'hémolymphe) après destruction des organes Y. Gen. comp. Endocrinol., 33, 35-40.

MOCQUARD J. P., BESSE G., JUCHAULT P., LEGRAND J.-J., MAISSIAT J., NOULIN G., 1971. Contribution à I'analyse du contrôle neurohumoral de la croissance, de la mue et de la physiologie sexuelle mâle et femelle chez l'Oniscoïde Ligia oceanica L. (Crustacé Isopode). Ann. Embryol. Morph., 4, 45-63.

NAGASAWA H., ISOGAI A., SUZUKI A., TAMURA S., ISHIZAKI H., 1979. Purification and properties of the prothoracicotropic hormone of the silkworm, Bombyx mori. Develop. Growth Differ., 21, $29-38$.

REIDENBACH J. M., 1971. Les mécanismes endocriniens dans le contrôle de la différenciation du sexe, la physiologie sexuelle et la mue chez le Crustacé Isopode marin : Idotea balthica (Pallas). Th. Doct. Etat, Nancy, CNRS n ${ }^{\circ}$ AO 4874, 335 pp.

VERNET G., 1976. Données actuelles sur le déterminisme de la mue chez les Crustacés. Année biol., 15, 155-188.

ZERBIB C., 1973. Contribution à l'étude ultrastructurale de l'ovocyte chez le Crustacé Amphipode Orchestia gammarella Pallas. C. R. Acad. Sci. Paris, sér. D, 277, 1209-1212.

ZERBIB C., 1976. Nature chimique des enclaves vitellines de l'ovocyte du Crustacé Amphipode Orchestia gammarellus (Pallas). Ann. Histochim., 21, 279-295.

ZERBIB C., 1980. Ultrastructural observation of oogenesis in the Crustacea Amphipoda Orchestia gammarellus (Pallas). Tissue Cell, 12, 47-62. 